\title{
Arnaud LESTREMAU, Donner sens au nom de personne dans le royaume anglo-saxon ( $\mathrm{X}^{e}-\mathrm{XI}$ siècles) : essai d'histoire sociale
}

\section{Pascal Chareille}

\section{(2penEdition}

\section{Journals}

Édition électronique

URL : https://journals.openedition.org/ccm/7478

DOI : $10.4000 / \mathrm{ccm} .7478$

ISSN : 2119-1026

\section{Éditeur}

Centre d'études supérieures de civilisation médiévale/Université de Poitiers

\section{Édition imprimée}

Date de publication : 1 juin 2021

Pagination : 171-174

ISBN : 978-2-490783-09-0

ISSN : 0007-9731

Référence électronique

Pascal Chareille, "Arnaud lestremau, Donner sens au nom de personne dans le royaume anglo-saxon ( $x^{e}$ $x l^{e}$ siècles) : essai d'histoire sociale », Cahiers de civilisation médiévale [En ligne], 254 | 2021, mis en ligne le 01 juin 2021, consulté le 10 décembre 2022. URL : http://journals.openedition.org/ccm/7478; DOI https://doi.org/10.4000/ccm.7478

\section{(c) $(1) \&$}

Creative Commons - Attribution - Pas d'Utilisation Commerciale - Pas de Modification 4.0 International - CC BY-NC-ND 4.0

https://creativecommons.org/licenses/by-nc-nd/4.0/ 
Arnaud Lestremau, Donner sens au nom de personne dans le royaume anglo-saxon ( $X^{e}-X I^{e}$ siècles) : essai d'histoire sociale, Turnhout, Brepols (Haut Moyen Âge, 35), 2018.

Version remaniée et augmentée d'une thèse de doctorat soutenue en 2013 et préparée sous la direction conjointe de Régine Le Jan et Pierre Bauduin, l'essai que propose Arnaud Lestremau est, à n'en pas douter, un ouvrage qui fera date sur un sujet où les travaux en langue française faisaient largement défaut. L'auteur le signale en introduction : «il n'existe pas, à ce jour, de grande étude abordant ensemble les questions de l'anthroponymie anglo-saxonne, des identités insulaires et, plus largement, des structures portantes de la société anglo-saxonne » (p. 15). C'est à cette tâche ardue qu'il s'est employé pour nous proposer une synthèse magistrale. Il se promène avec une aisance remarquable dans cette Angleterre anglo-saxonne pour nous faire découvrir, au prisme de l'anthroponymie, un monde dont il est aujourd'hui l'un des meilleurs et rares connaisseurs en France. Qu'il nous parle de la réorganisation de la cour d' Æthelred II ou du massacre de tous les Danois d'Angleterre le jour de la saint Brice en 1002 (p. 249), qu'il étudie le cas d'Orc (le Danois) (p. 244-247) pour démontrer que son nom est davantage marqueur d'étrangeté que d'ethnicité, qu'il appuie ses démonstrations sur des dénombrements pointus ou qu'il propose un bilan synthétique des diverses théories du nom propre (p. 56 et suiv.), A. Lestremau fait toujours preuve d'une très grande rigueur, dans la problématisation comme dans la démonstration, et son propos ne manque jamais d'ambition.

Parce que « les échelles auxquelles l'identité s'affirme sont nombreuses » (p. 248), il fait légitimement varier la focale en s'appuyant à la fois sur des analyses statistiques - au demeurant fort bien maîtrisées - à échelle « macro » sur tout ou partie des corpus qu'il a constitués, et sur des histoires familiales et individuelles qui lui permettent de donner de la chair à un propos qui, il faut l'avouer, est par moments quelque peu théorique et ardu. Il croise avec beaucoup de pertinence approches chronologiques, géographiques et sociales. Dans un style enlevé et impeccable, A. Lestremau montre avec habileté la spécificité de cette société anglo-saxonne soumise au métissage et au renouvellement de ses élites dirigeantes consécutifs aux vagues d'immigration successives.

D'une fort belle facture (346 pages dont 271 de texte à proprement parler, intégrant 37 graphiques, 15 cartes et 16 tableaux, mais aussi 10 stemmata présentés en annexe qui concernent les principaux groupes familiaux), l'ouvrage est d'une érudition singulière et ressemble plus à une œuvre de la maturité intellectuelle qu'à une production de jeunesse. On est saisi par une sorte de vertige en consultant la bibliographie proposée par A. Lestremau car il ne s'est pas contenté de lire les quelque 683 « sources secondaires » recensées; il les a, à l'évidence, parfaitement digérées. Toutes sont mobilisées de manière fort pertinente - comme en témoignent les 1737 notes de bas de page - et dans un souci d'exhaustivité qui frôle l'excès : A. Lestremau semble avoir cité tout ce qui pouvait l'être et il donne à son lecteur, qui découvre tout ce qu'il était possible de lire, quelques complexes... D'autant que certaines des références mentionnées dans les notes n'ont pas été intégrées dans la bibliographie finale sans qu'on puisse en saisir la raison (omissions volontaires ou accidentelles?). Parmi de nombreux exemples, Violaine Sebillote citée p. 28 note 59 n'est pas mentionnée dans les « sources secondaires $»$; Reinhard Wenskus, Patrick Wormald, Herwig Wolfram, Peter John Heather et Walter Pohl, cités en notes p. 29 ne le sont pas non plus; ni Ilona Opelt cité p. 54 note 13 ou Ingeborg Flugel p. 54 note 14; ni Maurizio Aceto p. 94 note 101 ou Roland Barthes p. 103, note 170, etc. La liste semble longue). Quoi qu'il en soit, ces références confirment la très grande érudition de l'auteur qui convoque philosophes, sociologues, anthropologues et linguistes au même titre que les historiens. Dans cet essai, la littérature médiévale côtoie les grammairiens; les théologiens les philologues; Ferdinand Tönnies, Émile Durkheim, Sigmund Freud et Marcel Mauss cohabitent avec Roland Barthes et Pierre Bourdieu. C'est bien à une forme "d'histoire totale " qu'A. Lestremau nous convie. Les compétences d'historien de l'auteur peuvent en outre s'appuyer sur des compétences de linguiste remarquables et fort utiles pour une lecture fine des noms dans une Angleterre pas encore anglo-normande mais déjà anglo-saxonne et anglo-scandinave : A. Lestremau maitrise le latin, le vieil anglais, le vieux germanique et le norrois ancien, sans parler des nombreuses langues modernes comme en témoignent les titres d'une bibliographie dont les références en langue française sont minoritaires. 
Les bornes chronologiques et le territoire couverts par l'étude sont clairs : de la fin du royaume viking d'York en 954 à la conquête normande de 1066; la Grande-Bretagne sans ses espaces celtiques (Paysde-Galle et Écosse). Un gros siècle, donc, pour ce qui est de la chronologie, mais A. Lestremau n'hésite pas à convier des auteurs de sources narratives dont les écrits sont postérieurs à 1066 ni à prendre en compte, le cas échéant, quelques documents utiles à son propos et produits au début du $\mathrm{X}^{\mathrm{e}} \mathrm{s}$. Au total, les sources mobilisées (recensées parmi les 254 « sources primaires » [p. 273-285] qui intègrent également les outils de travail) sont d'une grande variété et leur liste est, elle aussi, impressionnante. L'objectif est de compléter et de croiser les informations recueillies, mais aussi de «multiplier les points de vue et réduire les biais d'analyse » (p. 33). Les données anthroponymiques exploitées pour la période relèvent d'une logique d'accumulation (avec, là encore, la tentation de l'exhaustivité). Elles sont tirées de sources diplomatiques et fiscales - notamment de l'incontournable Domesday Book -, mais aussi de sources bibliques, homilétiques, nécrologiques, liturgiques, éducatives, hagiographiques, littéraires ou matérielles (épigraphiques ou numismatiques). A. Lestremau indique avoir recensé au total $926+327$ liens familiaux (p. 35, note 117), un ensemble de 2781 relations entre deux individus (p. 37, note 130), avoir exploité $722+123$ chartes ou autres documents diplomatiques (p. 40, tabl. 1), avoir procédé à « l'identification de près de 13000 personnes physiques ou morales dont 8057 sont nommées » (p. 47) et à celle de plus de 20000 monnaies (p. 50). Il me semble néanmoins qu'un tableau synthétique avec le nombre d'occurrences anthroponymiques recensées, le cas échéant de personnes et de noms, par type documentaire et par région, aurait été utile. A. Lestremau n'omet jamais de soumettre chacun de ces fonds à une critique documentaire rigoureuse pour en signaler les éventuelles spécificités, notamment parce que les sources disponibles sont possiblement différentes d'une période ou d'une région à l'autre.

Le plan de l'ouvrage témoigne d'une réflexion solidement structurée. Après une introduction générale qui annonce clairement les problèmes auxquels il s'agit de répondre, A. Lestremau propose six chapitres (décomposés en 3 ou 4 parties avec, pour chacun d'eux, une conclusion qui ramasse remarquablement le propos) parfaitement complémentaires.
Le chapitre 1 (« Le nom, substrat essentiel de l'identité », p. 21-52) permet à A. Lestremau de rappeler les fondements théoriques et méthodologiques de son propos. Il souligne la complexité de la notion d'identité (dont le nom est l'un des éléments) et ses multiples dimensions : identités individuelles, de groupe, construites, contextuelles, etc.

Le chapitre 2 ( "Nom et individuation », p. 53-81) propose une synthèse aboutie sur les théories savantes (notamment linguistiques et philosophiques) du nom. Objet socio-linguistique universel, le nom constitue « un espace où la transsubstantiation du social en langage s'opère visiblement » (p. 53). Les questions de l'individuation et de l'individualisation (avec, en creux, la question de la «naissance de l'individu »), et celle de l'étymologie ontologique des noms sont examinées dans ce chapitre.

Le chapitre 3 (« Nom et intégration sociale », p. 83-126) permet à A. Lestremau de montrer que les noms et les surnoms sont pensés et utilisés comme repères communs dans divers groupes sociaux (notamment au moment du baptême ou des rites funéraires) et qu'ils participent de la hiérarchisation de la société. Pour A. Lestremau, les surnoms n'ont pas été forgés pour distinguer les homonymes (p. 94) - c'est une thèse que Monique Bourin et moi-même défendons depuis longtemps. Il insiste aussi sur l'effet du type documentaire sur les surnoms (p. 98), confirmant là encore un point sur lequel l'enquête sur la genèse médiévale de l'anthroponymie moderne avait attiré l'attention (l'étude anthroponymique doit non seulement tenir compte du type documentaire mais aussi, au sein du matériau diplomatique, de la position dans l'acte, l'anthroponymie des témoins ou des signa se distinguant de celle des parties principales premières ou secondaires). La question de l'éventuel changement de nom des clercs et des moines (p. 100), celle des usages du passé autour de la question des pratiques mémorielles (p. 113) sont également abordées dans ce chapitre qui montre que l'appartenance à une communauté dévotionnelle est un « substrat important pour l'unification de la communauté politique anglo-saxonne » (p. 125).

Le chapitre 4 (« Nomen, famille et pouvoir", p. 127-178) aborde le problème complexe des relations entre noms et structures de parenté. La variabilité sociologique de ces structures et leur évolution dans le temps sont des questions qui agitent les médiévistes depuis des décennies, mais aussi, de manière 
plus large, de nombreux historiens (cf. par ex. le dernier numéro de L'Atelier du Centre de recherches historiques, 19 Bis, "Les Règles de la parenté, entre histoire et anthropologie ", 2018, en ligne), A. Lestremau montre que " les formes de transmission du nom sont plus répandues au sommet de l'échelle sociale » (p. 143) et, consécutivement, dans les espaces méridionaux du royaume, où résidaient la famille royale et une partie de l'aristocratie. Au total, « dans l'ensemble, la structure de la parenté anglo-saxonne est cognatique [avec une] tendance patrilinéaire [et] une lente maturation en faveur de la filiation en ligne masculine [...] [notamment] dans la famille royale, dont les tendances à la patrilinéarité et à l'unigéniture sont consubstantielles à la transmission de la couronne» (p. 157). La question des «noms programmatiques » ou légitimant des revendications de pouvoir est étudiée et illustrée (p. 166 et suiv.).

Le chapitre 5 (« Popularité des noms et structuration du corpus social », p. 179-210) permet à A. Lestremau de montrer que « bien que les palmarès fassent émerger des noms emblématiques [...], ces noms cachent un paysage onomastique varié, généralement riche et étendu, avec des taux d'homonymie assez faibles [...]. La créativité anthroponymique était toujours à l'œuvre au XI ${ }^{\mathrm{e}} \mathrm{s}$. » (p. 196).

Le chapitre 6 ( Peodisc Naman. Nom et appartenance ethnique », p. 211-261) aborde une question essentielle pour l'Angleterre anglo-saxonne car, si la langue est un critère objectif de définition des groupes, « la proximité linguistique entre noms anglo-saxons, norrois et continentaux $[\ldots]$ pose le problème de formes parfois impossibles à distinguer » (p. 258). Le succès des noms « acceptables par les deux cultures » est une preuve « de l'adaptation du stock anthroponymique au bilinguisme sociétal présent dans le royaume [qui] cadre parfaitement avec le développement d'une société de plus en plus métissée. » (p. 259) - une société que A. Lestremau, après Dawn M. Hadley, qualifie de "société anglo-scandinave ».

Dans sa conclusion générale (p. 263-271), A. Lestremau le précise lui-même : «l'idée majeure de cet ouvrage est que les identités sociales s'additionnent et se complètent [...] [Elles] sont en suspens dans le corps social et elles sont donc susceptibles de se combiner de façon variable et imprévisible » (p. 265). Dans la société anglo-saxonne, « le lien entre noms et identités ethniques est très subtil, conditionnel, susceptible de s'adapter aux besoins et il est impossible de le postuler d'une manière mécanique » (p. 271). Ici comme ailleurs, le surnom n'est pas la conséquence d'une hypothétique croissance de l'homonymie mais bien « un objet de communication politique et une marque de l'intégration dans une communauté d'interconnaissance » (p. 269).

L'étude anthroponymique a permis à A. Lestremau « de cerner avec une grande netteté l'impact du mouvement viking » (p. 264) en montrant l'attractivité des noms norrois au $\mathrm{XI}^{\mathrm{e}} \mathrm{s}$. ainsi que le succès des « noms ambigus mêlant influences scandinaves, anglo-saxonnes et continentales dans un stock de plus en plus unifié » (p. 264). Ces évolutions montrent un « renouvellement de la mode anthroponymique insulaire avec le déclin de quelques anciens noms (en celf- et en -rced) et une promotion des noms en god- et en -wine » (p. 264).

Cet essai est l'occasion de nuancer voire de corriger certains paradigmes de travaux antérieurs : «1) Cette période n'est pas livrée à l'absence de normes et aux incohérences qui découleraient des effets changeants de la mode, dans la mesure où l'inscription dans le champ de la parenté joue encore un rôle prépondérant $\mathrm{au} \mathrm{X}^{\mathrm{e}} \mathrm{s}$.; 2) le $\mathrm{XI}^{\mathrm{e}} \mathrm{s}$. ne constitue aucunement une période d'uniformisation des pratiques et d'homogénéisation du stock [...]; 3) les évolutions dans le champ de la parenté ne sont pas imputables à la présence de migrants danois, qui ont accéléré, tout au plus et à la marge, une dynamique qui était déjà en bonne part enclenchée quelques décennies avant leur prise de pouvoir » (p. 265).

S'il fallait formuler un regret, ce serait de ne pas avoir trouvé, associé à l'ouvrage, un cd-rom (où une URL) permettant au lecteur d'accéder à l'intégralité des bases de données constituées par A. Lestremau et sur lesquelles il s'est appuyé pour construire son propos. L'intérêt, pour l'ensemble des chercheurs qui s'intéressent au monde anglo-saxon, de cette métasource, même si elle s'appuie largement sur les données de la Prosopography of Anglo-Saxon England, ne fait aucun doute. Sa mise à disposition permettrait à chacun de pouvoir manipuler les données, d'examiner certains des choix méthodologiques qui ont été opérés, de produire possiblement des résultats nouveaux ou de vérifier certains de ceux établis par A. Lestemau. Il $\mathrm{y}$ a près d'un siècle, dans un propos visionnaire, Marc Bloch devinait dans l'étude des noms de personne 
un « instrument de sondage dont les résultats seront merveilleux, - lorsque nous en posséderons vraiment la technique » (Marc BLOCH, « Mr Karl Michaelsson, Études sur les noms de personne français d'après les rôles de taille parisiens [rôles de 1292, 12961300, 1313] [Note critique] », Annales d'histoire économique et sociale, IV-13, 1932, p. 67-69, DOI : 10.3406/ahess.1932.1586). L'essai d'A. Lestremau lui donne raison et démontre tout le profit qu'il est possible de tirer d'une maîtrise impeccable des concepts et des outils de l'anthroponymie.

Ce travail, qui apporte une contribution majeure à la réflexion sur l'influence scandinave en Angleterre, invite aussi les historiens à une vaste comparaison : comme l'Angleterre des $\mathrm{X}^{\mathrm{e}}$-XI ${ }^{\mathrm{e}} \mathrm{s}$., la Sicile (qui connaît elle aussi une conquête normande à la fin du $\mathrm{XI}^{\mathrm{e}} \mathrm{s}$.) et
l'Espagne sont, à la même époque, partagées politiquement et culturellement. Ces espaces sont des lieux de contacts entre plusieurs cultures : latino-chrétienne, normande, gréco-byzantine et arabo-islamique pour la Sicile; wisigothique, arabo-musulmane et latino-chrétienne pour l'Espagne. Observe-t-on des différences structurelles - et si oui lesquelles - quand on aborde les questions de l'anthroponymie, des identités et, plus largement, « des structures portantes » dans ces territoires à cette époque? Ce travail, éblouissant, ouvre la voie à cette réflexion comparative (voire structuraliste).

\author{
Pascal Chareille \\ EA 6298 - CeTHiS \\ Université de Tours
}

